\title{
A construction of an infinite family of 2 -arc transitive polygonal graphs of arbitrary even girth
}

\author{
Eric Swartz
}

Received: 24 June 2009 / Accepted: 26 April 2010 / Published online: 18 May 2010

(C) Springer Science+Business Media, LLC 2010

\begin{abstract}
A near-polygonal graph is a graph $\Gamma$ which has a set $\mathcal{C}$ of $m$-cycles for some positive integer $m$ such that each 2-path of $\Gamma$ is contained in exactly one cycle in $\mathcal{C}$. If $m$ is the girth of $\Gamma$, then the graph is called polygonal. We provide a construction of an infinite family of polygonal graphs of arbitrary even girth with 2-arc transitive automorphism groups, showing that there are infinitely many 2-arc transitive polygonal graphs of every girth.
\end{abstract}

Keywords Algebraic graph theory $\cdot$ Polygonal graph $\cdot$ 2-arc transitive graph

\section{Introduction}

Let $\Gamma$ be a graph. For a positive integer $l$, an $l$-walk of $\Gamma$ is a sequence of vertices $\left(\alpha_{0}, \alpha_{1}, \ldots, \alpha_{l}\right)$ such that $\alpha_{i}$ is adjacent to $\alpha_{i+1}$ for $0 \leq i \leq l-1$. If in addition $\alpha_{i-1} \neq \alpha_{i+1}$ for $1 \leq i \leq l-1$, then an $l$-walk is called an $l$-arc; while if further all the $\alpha_{i}$ are distinct, then the $l$-arc is called an $l$-dipath (directed path). The identification of an $l$-dipath $\left(\alpha_{0}, \alpha_{1}, \ldots, \alpha_{l}\right)$ and its reverse $\left(\alpha_{l}, \ldots, \alpha_{1}, \alpha_{0}\right)$ is called an $l$-path, and denoted by $\left[\alpha_{0}, \alpha_{1}, \ldots, \alpha_{l}\right]$. An $m$-cycle is an $(m-1)$-path $\left[\alpha_{1}, \ldots, \alpha_{m}\right]$ such that $\alpha_{m}$ is adjacent to $\alpha_{1}$.

We call $\Gamma$ a near-polygonal graph if there exists a number $m$ and a collection $\mathcal{C}$ of $m$-cycles in $\Gamma$ such that each 2-path of $\Gamma$ is contained in exactly one cycle in $\mathcal{C}$. If $m$ is the girth $g(\Gamma)$ of $\Gamma$, then the graph is called polygonal. The notions of polygonal and near-polygonal graphs were invented by Perkel (see, for example, [2]).

Before [4], the only examples of 2-arc transitive polygonal graphs with arbitrarily large valency had girth no larger than seven, and the 2-arc transitive polygonal graph

E. Swartz ( $\varangle)$

Department of Mathematics, The Ohio State University, Columbus, OH 43210, USA

e-mail: eswartz@math.ohio-state.edu 
with largest girth had valency five and girth twenty-three (in fact, even with no restrictions on the automorphism group, there were no examples of polygonal graphs with odd girth greater than twenty-three) [3]. In [4] infinite families of polygonal graphs of arbitrary odd girth were constructed.

The main purpose of this paper is to construct 2-arc transitive polygonal graphs of arbitrarily large valency and girth. We will prove the following results.

Theorem 1.1 There are 2-arc transitive polygonal graphs of arbitrarily large valency and girth $m$ for all even $m \geq 4$.

Corollary 1.2 There are infinitely many 2-arc transitive polygonal graphs of girth $m$ for all $m \geq 3$, and there are 2-arc transitive polygonal graphs of arbitrarily large valency for each $m$.

\section{Notation and preliminary results}

The construction in this paper is a generalization of that in [1] and so relies heavily upon its notation and results. As in [1], an $(l, m)$-path-cycle cover of a graph $\Gamma$ is a set $\mathcal{C}$ of $m$-cycles such that each $l$-path of $\Gamma$ is covered by at least one cycle in $\mathcal{C}$; sometimes an $(l, m)$-path-cycle cover is simply called an $(l, m)$-cover. If in addition every $l$-path of $\Gamma$ lies in a constant number $\lambda$ of cycles of $\mathcal{C}$, then $\mathcal{C}$ is called a regular $\lambda$ - $(l, m)$-cover, or simply called a $\lambda$ - $(l, m)$-cover. Hence near-polygonal graphs are the graphs that have a $1-(2, m)$-cover for some $m$.

For a graph $\Gamma$ and a group $G \leq \operatorname{Aut}(\Gamma)$, an $(l, m)$-cover $\mathcal{C}$ is called $G$-symmetrical if $\mathcal{C}=\left\{C_{1}, C_{2}, \ldots, C_{n}\right\}$ is such that

(i) The restriction $\left.G\right|_{C_{i}}$ of $G$ to each $C_{i}$ contains all rotations of $C_{i}$.

(ii) $G$ induces a transitive action on $\mathcal{C}$.

There are two possibilities for $\left.G\right|_{C_{i}}$ to contain all rotations of $C_{i}$, namely $\left.G\right|_{C_{i}} \cong$ $\mathbb{Z}_{m}$ or $\left.G\right|_{C_{i}} \cong \mathrm{D}_{2 m}$. The corresponding symmetrical covers will be called $G$-rotary or $G$-dihedral, respectively. For a positive integer $l$, a graph $\Gamma$ is called $(G, l)$-arc transitive, $(G, l)$-dipath transitive, or $(G, l)$-path transitive if $G$ acts transitively on $l$ arcs, $l$-dipaths, or $l$-paths of $\Gamma$, respectively. In the case of dipath and path transitivity, we also require that $l$-dipaths or $l$-paths exist in $\Gamma$, respectively.

We now present the basis for the construction of near-polygonal graphs in [1].

Lemma 2.1 [1, Lemma 1.1] Let $\Gamma$ be a regular graph of valency at least three, let $G \leq \operatorname{Aut}(\Gamma)$, and let $l \geq 1$ be an integer. Then $(\mathrm{a}) \Rightarrow(\mathrm{b}) \Rightarrow(\mathrm{c}) \Rightarrow$ (d) holds for the following four statements (a)-(d).

(a) $\Gamma$ has a $G$-dihedral $(l, m)$-cover for some $m \geq 3$.

(b) $\Gamma$ is $(G, l)$-dipath transitive.

(c) $\Gamma$ has a $G$-rotary $(l, m)$-cover for some $m \geq 3$.

(d) $\Gamma$ is $(G, l)$-path transitive.

Moreover, if $\Gamma$ has a $G$-dihedral $(l, m)$-cover $\mathcal{C}$ and $G$ acts sharply transitively on the l-dipaths in $\Gamma$, then $\mathcal{C}$ is a 1- $(l, m)$-cover. 
We will use Lemma 2.1 in conjunction with the following method for construction.

Construction 2.2 [1, Construction 1.1] Let $\left(\alpha_{0}, \ldots, \alpha_{l}\right)$ and $\left(\alpha_{1}, \ldots, \alpha_{l}, \alpha_{l+1}\right)$ be l-dipaths in a graph $\Gamma$ (allowing that $\left.\alpha_{0}=\alpha_{l+1}\right)$, let $G \leq \operatorname{Aut}(\Gamma)$, and suppose that there exists $g \in G$ such that $\alpha_{i}^{g}=\alpha_{i+1}$ for $0 \leq i \leq l$. Let $C$ be the cycle generated by the vertices $\alpha_{0}^{\langle g\rangle}$, and let $\mathcal{C}=C^{G}$.

We shall refer to the method described in Construction 2.2 as spinning an l-dipath. In order to apply Lemma 2.1, we need to ensure that a target group $G$ occurs as a group of automorphisms of some graph $\Gamma$. That goal can be achieved by defining $\Gamma$ as a coset graph (also called orbital graph), as described below.

For a group $G$ and a subgroup $H<G$, denote by $[G: H]$ the set of right cosets of $H$ in $G$. For an element $g \in G \backslash H$ with $g^{2} \in H$, the coset graph $\Gamma:=$ $\operatorname{Cos}(G, H, H g H)$ is defined as the graph with vertex set $[G: H]$ such that two vertices $H x$ and $H y$ are adjacent if and only if $y x^{-1} \in H g H$. Observe that from the condition $g^{2} \in H$ it follows that $H g H=H^{-1} H$ and $H x$ and $H y$ are adjacent if and only if $H y$ and $H x$ are adjacent, implying that $\Gamma$ is undirected. Denote by $\alpha$ the vertex $H$ of $\Gamma$ and by $\beta$ the vertex $\alpha^{g}=H g$. Note that $\beta^{g}=\alpha^{g^{2}}=\alpha, G_{\alpha}=H$, $G_{\beta}=H^{g}$, and $G_{\alpha \beta}=H \cap H^{g}$. The neighbor set $N_{\Gamma}(\alpha)$ of $\alpha$ consists of the cosets in $H g H$, and the valency of $\alpha$ is the index $\left|G_{\alpha}: G_{\alpha \beta}\right|$.

For constructing near-polygonal graphs as in [1], we want to spin a 2-dipath $(\gamma, \alpha, \beta)$. The spinning element can be described easily in terms of the coset graph.

Lemma 2.3 [1, Lemma 2.3] For a coset graph $\Gamma=\operatorname{Cos}(G, H, H g H)$ with $g^{2} \in H$, let $\alpha=H$ and $\beta=\alpha^{g}$. Then an element $f \in G$ maps $\alpha$ to $\beta$ if and only if $f \in\left(G_{\alpha}\right) g$. Furthermore, for $f \in G$ such that $\alpha^{f}=\beta$, we have that $\beta \neq \alpha^{f^{-1}}$ if and only if $f \in\left(G_{\alpha}\right) g \backslash\left(G_{\alpha \beta}\right) g$.

Finally, we will use the following well-known lemma as stated in [1].

Lemma 2.4 [1, Lemma 2.4] Let $\Gamma$ be a graph, and let $G \leq \operatorname{Aut}(\Gamma)$ be transitive on the vertex set of $\Gamma$. Then $\Gamma$ is $(G, 2)$-dipath transitive if and only if $G_{\alpha}$ acts 2-transitively on $N_{\Gamma}(\alpha)$; furthermore, $\Gamma$ is sharply $(G, 2)$-dipath transitive if and only if $G_{\alpha}$ acts sharply 2-transitively on $N_{\Gamma}(\alpha)$.

\section{A family of polygonal graphs with cycles of a fixed even length}

We will use essentially the same construction as in [4], where a family of polygonal graphs with cycles of a fixed odd length was produced. Let $m=2 k+2, k \in \mathbb{N}$.

We define $G$ to be the direct product of $k$ copies of $\operatorname{PGL}(2, q)$, where $q$ is a prime power, i.e., $G:=\prod_{i=1}^{k} \operatorname{PGL}(2, q)$. The elements of $\operatorname{PGL}(2, q)$ can be identified with equivalence classes of $2 \times 2$ invertible matrices over the field $\operatorname{GF}(q)$, with two matrices equivalent if and only if they are scalar multiples of each other. 
With a slight abuse of notation, we shall write that the matrices themselves are elements of PGL $(2, q)$, and that the elements of $G$ are $k$-tuples of matrices. We identify $H \cong A G L(1, q)$ with the set of (equivalence classes of) lower triangular matrices $\left\{\left[\begin{array}{ll}a & 0 \\ b & c\end{array}\right]: a, b, c \in \operatorname{GF}(q), a c \neq 0\right\}$ and define $\bar{H}:=\operatorname{Diag}\left(\prod_{i=1}^{k} A G L(1, q)\right)=$ $\{(h, h, \ldots, h): h \in H\} \leq G$.

For $a \in \mathrm{GF}(q)$, let $\bar{p}(a):=\left[\begin{array}{ll}1 & 0 \\ a & 1\end{array}\right] \in H$ and $\bar{p}(a):=(p(a), p(a), \ldots, p(a)) \in \bar{H}$. Moreover, if $a \neq 0$, then let $d(a):=\left[\begin{array}{ll}a & 0 \\ 0 & 1\end{array}\right] \in H$ and $\bar{d}(a):=(d(a), d(a), \ldots, d(a)) \in$ $\bar{H}$. Let $P=\{p(a): a \in \mathrm{GF}(q)\}, \bar{P}=\{\bar{p}(a): a \in \mathrm{GF}(q)\}, D=\left\{d(a): a \in \mathrm{GF}(q)^{*}\right\}$, and $\bar{D}=\left\{\bar{d}(a): a \in \mathrm{GF}(q)^{*}\right\}$. Then $H=P D, \bar{H}=\overline{P D}, P \triangleleft H$, and $\bar{P} \triangleleft \bar{H}$.

For $y \in \operatorname{GF}(q)^{*}$, let $g(y)=\left[\begin{array}{cc}0 & y \\ -1 & 0\end{array}\right]$. Then, for $g=g\left(y_{1}, y_{2}, \ldots, y_{k}\right):=\left(g\left(y_{1}\right)\right.$, $\left.g\left(y_{2}\right), \ldots, g\left(y_{k}\right)\right) \in G$, we have $g^{2}=1 \in \bar{H}$, so we can define the coset graph $\Gamma=$ $\Gamma\left(y_{1}, y_{2}, \ldots, y_{k}\right):=\operatorname{Cos}(G, \bar{H}, \bar{H} g \bar{H})$. Let $\alpha$ denote the vertex $\bar{H}$, and let $\beta$ denote the vertex $\bar{H} g$. First we determine the number of vertices, the valency, and bound the number of components of $\Gamma$. The number of vertices is

$$
|G: \bar{H}|=|G| /|\bar{H}|=q^{k-1}(q-1)^{k-1}(q+1)^{k} .
$$

For any $\bar{d} \in \bar{D}$, we have $\bar{d}^{g}=\bar{d}^{-1}$, so $G_{\alpha \beta}=\bar{H} \cap \bar{H}^{g} \geq \bar{D}$. Since $\bar{D}$ is a maximal subgroup of $\bar{H}$, we must have equality here, and so the valency of $\Gamma$ is

$$
\left|G_{\alpha}: G_{\alpha \beta}\right|=|\bar{H}: \bar{D}|=q \text {. }
$$

For a vertex $\delta$ of $\Gamma$, let $W^{(r)}(\delta)$ denote the set of vertices reachable by an $r$-long walk from $\delta$. Then we have the following, which has the same proof as [1, Lemma 3.1]:

Lemma 3.1 $W^{(r)}(\alpha)=\bar{H} g \bar{P} g \bar{P} \cdots g \bar{P}$ (r iterations of $\left.g \bar{P}\right)$.

Moreover, we have the following results from [4]:

Lemma 3.2 [4, Lemma 3.2] Let $y_{1}, y_{2}, \ldots, y_{k}$ be distinct elements of $\mathrm{GF}(q)^{*}$. Then $\Gamma$ has at most $2^{k-1}$ components.

Lemma 3.3 [4, Lemma 3.3] For all $y_{1}, y_{2}, \ldots, y_{k} \in \mathrm{GF}(q)^{*}$, the graph $\Gamma$ is nearpolygonal.

Lemma 3.4 [4, Lemma 3.5] Let $q \equiv \pm 1(\bmod m)$, and let $\zeta=\zeta_{m}$ be a primitive $m$ th root of unity in $\operatorname{GF}\left(q^{2}\right)$. Then, if $y_{i}:=\zeta^{i}+\zeta^{m-i}+2 \in \mathrm{GF}(q)^{*}$ for $1 \leq i \leq k$, all the $y_{i}$ are distinct, and the graph $\Gamma=\Gamma\left(y_{1}, \ldots, y_{k}\right)$ has a 1-(2,m)-cover.

We now proceed to show that the girth of the graph $\Gamma$ we have just constructed is at least $m$. We define

$$
r^{(l)}(y):=\prod_{i=1}^{l} g(y) p\left(a_{i}\right)
$$


where $y \in \operatorname{GF}(q)^{*}$, and for all $i, a_{i} \in \operatorname{GF}(q)^{*}$. Note that we view $r^{(l)}(y)$ as a function of $y$ given arbitrary fixed units $a_{1}, \ldots, a_{l} \in \mathrm{GF}(q)^{*}$. The following is an extension of [4, Lemma 3.6]:

Lemma 3.5 If $r^{(l)}(y)=\left[\begin{array}{c}r_{11}^{(l)}(y) r_{12}^{(l)}(y) \\ r_{21}^{(l)}(y)\end{array} r_{22}^{(l)}(y)\right]$, then for all $l \geq 1$,

(a) $r_{12}^{(l)}(y)=y r_{11}^{(l-1)}(y)$, where we define $r^{(0)}(y)=I_{2}$, the $2 \times 2$ identity matrix.

(b) $\operatorname{deg}\left(r_{11}^{(l)}(y)\right)=l$.

(c) $r_{11}^{(l)}(y)=y^{\left\lceil\frac{l}{2}\right\rceil} s_{l}(y)$, where $s_{l}(y)$ is a degree $\left\lfloor\frac{l}{2}\right\rfloor$ polynomial in $y$ with leading coefficient $a_{1} a_{2} \cdots a_{l}$.

(d)

$$
s_{l}(y)= \begin{cases}y a_{l} s_{l-1}(y)-s_{l-2}(y), & \text { l even, } \\ a_{l} s_{l-1}(y)-s_{l-2}(y), & \text { lodd, }\end{cases}
$$

where we define $s_{0}(y)=1$ and $s_{-1}(y)=0$.

(e) For all $l \geq 2$, the coefficient of $y^{\left.L^{\frac{l}{2}}\right\rfloor-1}$ in $s_{l}(y)$, denoted by $\mathcal{T}_{l}$, is the negative of the sum of $l-1$ terms, each of which is the product of $l-2$ different $a_{i},\left\lceil\frac{l-2}{2}\right\rceil$ of which are odd $i$.

(f) The constant term of $s_{l}(y)$, denoted by $\mathcal{K}_{l}$, is $(-1)^{\frac{l}{2}}$ for l even and $(-1)^{\frac{l-1}{2}}\left(a_{1}+\right.$ $\left.a_{3}+a_{5}+\cdots+a_{l}\right)$ for $l$ odd.

(g) The linear term of $s_{l}(y)$ for l even has coefficient $\mathcal{S}_{l}$, where $\mathcal{S}_{l}$ is the product of $(-1)^{\frac{l}{2}+1}$ and the sum of $\frac{\left(\frac{l}{2}\right)\left(\frac{l}{2}+1\right)}{2}$ terms of the form $a_{i} a_{j}$, where $i$ is odd and $j$ is even.

(h) $r_{22}^{(l)}=y r_{21}^{(l-1)}(y)$.

(i) $\operatorname{deg}\left(r_{21}^{(l)}(y)\right)=l-1$.

(j) $r_{21}^{(l)}(y)=y^{\left\lceil\frac{l-1}{2}\right\rceil} t_{l}(y)$, where $t_{l}(y)$ is a degree $\left\lfloor\frac{l-1}{2}\right\rfloor$ polynomial in $y$ with leading coefficient $-a_{2} a_{3} \cdots a_{l}$.

(k)

$$
t_{l}(y)= \begin{cases}y a_{l} t_{l-1}(y)-t_{l-2}(y), & \text { lodd }, \\ a_{l} t_{l-1}(y)-t_{l-2}(y), & \text { l even } .\end{cases}
$$

(1) The constant term of $t_{l}(y)$, denoted by $\mathcal{K}_{l}^{\prime}$, is $(-1)^{\frac{l+1}{2}}$ for l odd and $(-1)^{\frac{l}{2}}\left(a_{2}+\right.$ $\left.a_{4}+a_{6}+\cdots a_{l}\right)$ for l even.

Proof Parts (a)-(c) were proved in [4].

We note first that $g(y) p(a)=\left[\begin{array}{cc}y a & y \\ -1 & 0\end{array}\right]$, and so:

$$
\begin{gathered}
r^{(1)}(y)=\left[\begin{array}{cc}
y a_{1} & y \\
-1 & 0
\end{array}\right], \\
r^{(2)}(y)=\left[\begin{array}{cc}
y\left(y a_{1} a_{2}-1\right) & y\left(y a_{1}\right) \\
-y a_{2} & -y
\end{array}\right],
\end{gathered}
$$




$$
\begin{aligned}
r^{(l)}(y) & =r^{(l-1)}(y) \cdot\left[\begin{array}{cc}
y a_{l} & y \\
-1 & 0
\end{array}\right] \\
& =\left[\begin{array}{ll}
r_{11}^{(l-1)} & r_{12}^{(l-1)} \\
r_{21}^{(l-1)} & r_{22}^{(l-1)}
\end{array}\right] \cdot\left[\begin{array}{cc}
y a_{l} & y \\
-1 & 0
\end{array}\right] \\
& =\left[\begin{array}{ll}
y a_{l} r_{11}^{(l-1)}(y)-r_{12}^{(l-1)}(y) & y r_{11}^{(l-1)}(y) \\
y a_{l} r_{21}^{(l-1)}(y)-r_{22}^{(l-1)}(y) & y r_{21}^{(l-1)}(y)
\end{array}\right] .
\end{aligned}
$$

For (d), we note that $s_{1}(y)=a_{1}, s_{2}(y)=y a_{1} a_{2}-1$ and proceed by induction. We assume the result holds for $n=l-2, l-1$. Then using (a), (c), and (3.3), we find that

$$
\begin{aligned}
r_{11}^{(l)}(y) & =y^{\left\lceil\frac{l}{2}\right\rceil} s_{l}(y) \\
& =y a_{l} r_{11}^{(l-1)}(y)-r_{12}^{(l-1)}(y) \\
& =y a_{l}\left(y^{\left\lceil\frac{l-1}{2}\right\rceil} s_{l-1}(y)\right)-y\left(y^{\left\lceil\frac{l-2}{2}\right\rceil} s_{l-2}(y)\right) .
\end{aligned}
$$

Checking the cases for $l$ odd and $l$ even distinctly yields the desired result.

For (e), we proceed by induction. Given that $s_{2}(y)=y a_{1} a_{2}-1$ and $s_{3}(y)=$ $y a_{1} a_{2} a_{3}-\left(a_{1}+a_{3}\right)$, we have our base case. Assume that the result holds for $n=l-1$. By (d) and (c),

$$
\mathcal{T}_{l}=a_{l}\left(\mathcal{T}_{l-1}\right)-a_{1} a_{2} \cdots a_{l-2}
$$

so we have $(l-2)+1=(l-1)$ terms, each of which is the negative of the product of $(l-2)$ different $a_{i},\left\lceil\frac{l-2}{2}\right\rceil$ are odd $i$.

For (f), we proceed by induction with base cases $s_{1}(y)=a_{1}, s_{2}(y)=y a_{1} a_{2}-1$, $s_{3}(y)=y a_{1} a_{2} a_{3}-\left(a_{1}+a_{3}\right)$, and assume that the result is true for $n=l-2, l-1$. We note by (d) that for $l$ even,

$$
\mathcal{K}_{l}=-\mathcal{K}_{l-2},
$$

proving the even case, and for $l$ odd,

$$
\begin{aligned}
\mathcal{K}_{l} & =a_{l} \mathcal{K}_{l-1}-\mathcal{K}_{l-2} \\
& =a_{l}(-1)^{\frac{l-1}{2}}-(-1)^{\frac{l-3}{2}}\left(a_{1}+a_{3}+\cdots+a_{l-2}\right), \\
& =(-1)^{\frac{l-1}{2}}\left(a_{1}+a_{3}+\cdots+a_{l}\right)
\end{aligned}
$$

which proves the result.

For $(\mathrm{g})$, we again proceed by induction. We have base case $s_{2}(y)=y a_{1} a_{2}-1$. We now assume that the result is true for $n=l-2$ even. By (d),

$$
\mathcal{S}_{l}=-\mathcal{S}_{l-2}+a_{l} \mathcal{K}_{l-1}
$$

Using (f), this gives the desired result.

For (h), we again use induction and refer simply to (3.3). 
For (i) and (j), we use induction and refer to (3.1) and (3.2) as our base cases. (3.3) gives us

$$
\begin{aligned}
r_{21}^{(l)}(y) & =y a_{l} r_{21}^{(l-1)}(y)-r_{22}^{(l-1)}(y) \\
& =y a_{l} r_{21}^{(l-1)}(y)-y r_{21}^{(l-2)}(y),
\end{aligned}
$$

immediately using the result from (h).

To prove (i), we proceed by induction and assume that it is true for all $n<l$. $\mathrm{By}(3.4), r_{21}^{(l)}(y)$ is by inductive hypothesis the difference of a degree $l-1$ polynomial in $y$ and a degree $l-2$ polynomial in $y$ and hence is itself a degree $l$ polynomial in $y$.

To prove (j), we again proceed by induction on $l$ and assume that it holds for all $n<l$. Applying our inductive hypothesis,

$$
\begin{aligned}
r_{21}^{(l)}(y) & =y a_{l} r_{21}^{(l-1)}(y)-y r_{21}^{(l-2)}(y) \\
& =y a_{l} \cdot y^{\left\lceil\frac{l-2}{2}\right\rceil} t_{l-1}(y)-y \cdot y^{\left\lceil\frac{l-3}{2}\right\rceil} t_{l-2}(y) \\
& =y^{\left\lceil\frac{l-1}{2}\right\rceil}\left(y^{\left\lceil\frac{l}{2}\right\rceil-\left\lceil\frac{l-1}{2}\right\rceil} a_{l} t_{l-1}(y)-t_{l-2}(y)\right) .
\end{aligned}
$$

Since $t_{l-1}(y)$ has leading coefficient $-a_{2} a_{3} \cdots a_{l-1}$, we now see that $t_{l}(y)$ has leading coefficient $-a_{2} a_{3} \cdots a_{l-1} a_{l}$, and

$$
\begin{aligned}
\operatorname{deg}\left(t_{l}(y)\right) & =\operatorname{deg}\left(r_{21}^{(l)}(y)\right)-\left\lceil\frac{l-1}{2}\right\rceil \\
& =(l-1)-\left\lceil\frac{l-1}{2}\right\rceil=\left\lfloor\frac{l-1}{2}\right\rfloor,
\end{aligned}
$$

as desired.

For $(\mathrm{k})$, we again use induction with base cases $t_{1}(y)=-1, t_{2}(y)=-a_{2}, t_{3}(y)=$ $y\left(-a_{2} a_{3}\right)+1$. We assume that the result is true for all $n<l$, and using (h), (i), and (3.3), we find that

$$
\begin{aligned}
r_{21}^{(l)}(y) & =y a_{l} r_{21}^{(l-1)}(y)-r_{22}^{(l-1)}(y) \\
& =y a_{l}\left(y^{\left\lceil\frac{l-2}{2}\right\rceil} t_{l-1}(y)\right)-y\left(y^{\left\lceil\frac{l-3}{2}\right\rceil} t_{l-2}(y)\right) .
\end{aligned}
$$

Checking the cases for $l$ odd and $l$ even distinctly yields the desired result.

For (1), we proceed by induction with base cases $t_{1}(y)=-1, t_{2}(y)=-a_{2}, t_{3}(y)=$ $y\left(-a_{2} a_{3}\right)+1, t_{4}(y)=y^{2}\left(-a_{2} a_{3} a_{4}\right)+\left(a_{2}+a_{4}\right)$ and assume that the result is true for $n<l$. We note by (k) that for $l$ odd,

$$
\mathcal{K}_{l}^{\prime}=-\mathcal{K}_{l-2}^{\prime},
$$


proving the odd case, and for $l$ even,

$$
\begin{aligned}
\mathcal{K}_{l}^{\prime} & =a_{l} \mathcal{K}_{l-1}^{\prime}-\mathcal{K}_{l-2}^{\prime} \\
& =a_{l}(-1)^{\frac{l}{2}}-(-1)^{\frac{l-2}{2}}\left(a_{2}+a_{4}+\cdots+a_{l-2}\right) \\
& =(-1)^{\frac{l}{2}}\left(a_{2}+a_{4}+\cdots+a_{l}\right),
\end{aligned}
$$

which proves the result and the lemma.

With the following lemma, we are almost able to show that the graphs are polygonal:

Lemma 3.6 Let $m=2 k+2$ for some $k \in \mathbb{N}, q$ a prime power such that $q \equiv \pm 1$ $(\bmod m)$, and let $\Gamma$ be a near-polygonal graph with special cycles of length $m$ as constructed in Lemma 3.4. Then the girth of $\Gamma$ is at least $m-1$.

Proof The following is analogous to the proof of [4, Theorem 1.1]. It is included for the sake of completeness and to give a flavor for what further work will need to be done.

If $m=2 k+2$ for some $k \in \mathbb{N}, q \equiv \pm 1(\bmod m), \zeta$ is a primitive $m$ th root of unity in $\operatorname{GF}\left(q^{2}\right)$, and $y_{i}:=\zeta^{i}+\zeta^{m-i}+2$, then, by Lemma 3.4, $\Gamma=\Gamma\left(y_{1}, \ldots, y_{k}\right)$ is a near-polygonal graph with cycles of length $m$. Assume that $\Gamma$ has girth $n<$ $m-1$. This means that $\alpha \in W^{(n)}(\alpha)=\bar{H} \prod_{i=1}^{n} g \bar{P}$ by Lemma 3.1 and that there was no "backtracking" along our $n$-walk, i.e., we have an $(n-1)$-dipath $\left(\delta_{0}=\right.$ $\left.\alpha, \delta_{1}, \ldots, \delta_{n-1}\right)$ with $\delta_{n-1}$ adjacent to $\alpha$ and no shorter dipaths from $\alpha$ to itself in $\Gamma$. Thus $\bar{H} \prod_{i=1}^{n} g \bar{P}=\bar{H}$, which means that there exist $a_{1}, a_{2}, \ldots, a_{n} \in \mathrm{GF}(q)$ such that $\prod_{i=1}^{n} g \bar{p}\left(a_{i}\right) \in \bar{H}$, which in turn implies that $\prod_{i=1}^{n} g\left(y_{j}\right) p\left(a_{i}\right)$ is a lower diagonal matrix for each $1 \leq j \leq k$. Note that for $1 \leq t \leq(n-1), a_{t} \neq 0$, since otherwise

$$
\begin{aligned}
\prod_{i=1}^{n} g \bar{p}\left(a_{i}\right) & =g \bar{p}\left(a_{1}\right) \cdots g \bar{p}\left(a_{t-1}\right) g 1 g \bar{p}\left(a_{t+1}\right) \cdots g \bar{p}\left(a_{n}\right) \\
& =g \bar{p}\left(a_{1}\right) \cdots g \bar{p}\left(a_{t-1}+a_{t+1}\right) \cdots g \bar{p}\left(a_{n}\right),
\end{aligned}
$$

which is at most a nontrivial $(n-1)$-walk from $\alpha$, contradicting our assumption of girth $n$. Note that

$$
\begin{aligned}
\prod_{i=1}^{n} g(y) p\left(a_{i}\right) & =r^{(n-1)}(y) \cdot g(y) p\left(a_{n}\right) \\
& =\left[\begin{array}{ll}
r_{11}^{(n-1)} & r_{12}^{(n-1)} \\
r_{21}^{(n-1)} & r_{22}^{(n-1)}
\end{array}\right] \cdot\left[\begin{array}{cc}
y a_{n} & y \\
-1 & 0
\end{array}\right] \\
& =\left[\begin{array}{ll}
y a_{n} r_{11}^{(n-1)}(y)-r_{12}^{(n-1)}(y) & y r_{11}^{(n-1)}(y) \\
y a_{n} r_{21}^{(n-1)}(y)-r_{22}^{(n-1)}(y) & y r_{21}^{(n-1)}(y)
\end{array}\right],
\end{aligned}
$$


and so

$$
\begin{aligned}
\prod_{i=1}^{n} g \bar{p}\left(a_{i}\right)= & \left(\left[\begin{array}{ll}
y_{1} a_{n} r_{11}^{(n-1)}\left(y_{1}\right)-r_{12}^{(n-1)}\left(y_{1}\right) & y_{1} r_{11}^{(n-1)}\left(y_{1}\right) \\
y_{1} a_{n} r_{21}^{(n-1)}\left(y_{1}\right)-r_{22}^{(n-1)}\left(y_{1}\right) & y_{1} r_{21}^{(n-1)}\left(y_{1}\right)
\end{array}\right], \ldots,\right. \\
& {\left.\left[\begin{array}{ll}
y_{k} a_{n} r_{11}^{(n-1)}\left(y_{k}\right)-r_{12}^{(n-1)}\left(y_{k}\right) & y_{k} r_{11}^{(n-1)}\left(y_{k}\right) \\
y_{k} a_{n} r_{21}^{(n-1)}\left(y_{k}\right)-r_{22}^{(n-1)}\left(y_{k}\right) & y_{k} r_{21}^{(n-1)}\left(y_{k}\right)
\end{array}\right]\right), }
\end{aligned}
$$

and by Lemma 3.5(c)

$$
y r_{11}^{(n-1)}(y)=y \cdot y^{\left\lceil\frac{n-1}{2}\right\rceil} s_{n-1}(y) .
$$

Hence each $y_{i}$ is a root of the right-hand side of (3.5) in $\operatorname{GF}(q)$ since $\prod_{i=1}^{n} g \bar{p}\left(a_{i}\right)$ is lower diagonal. We know from Lemma 3.4 that for all $i, y_{i} \neq 0$. Thus each $y_{i}$ for $1 \leq i \leq k$ must be a root of $s_{n-1}(y)$ in $\operatorname{GF}(q)$ if the girth of $\Gamma$ is $n$. The $y_{i}$ are all distinct, and so this implies that $\operatorname{deg}\left(s_{n-1}(y)\right) \geq k$. But, by Lemma 3.5(c),

$$
\operatorname{deg}\left(s_{n-1}(y)\right)=\left\lfloor\frac{n-1}{2}\right\rfloor \leq\left\lfloor\frac{2 k-1}{2}\right\rfloor=k-1
$$

since $n<m-1$, a contradiction. Therefore the girth of $\Gamma$ is at least $m-1$.

For the graphs of odd girth constructed in [4], the techniques used in the above lemma were enough to show that the graphs were actually polygonal. Here, however, we need the following additional theorem:

Theorem 3.7 Let $m=2 k+2$ for some $k \in \mathbb{N}$, $p$ a prime, $q=p^{n}$ for some $n \in \mathbb{N}$, $q \equiv \pm 1(\bmod m)$, and let $\Gamma$ be a near-polygonal graph with special cycles of length $m$ as constructed in Lemma 3.4. If $\Gamma$ has girth $(m-1)$, then either

(i) $p \mid(k-1)$ and $p \mid\left(2^{k-1}-1\right)$.

(ii) $p \mid(4 k+3)$ and $p \mid\left(5^{k}-4 \cdot 3^{k}\right)$.

Proof We must look precisely at the case where we have a cycle of length $m-1$. Since we have a 2 -arc transitive automorphism group and $\Gamma$ is $(G, 2)$-dipath transitive, we may assume that our cycle of length $m-1$ includes $\left[\gamma=\bar{H} f^{-1}, \alpha=\bar{H}, \beta=\right.$ $\bar{H} g$ ], i.e., we may assume that $\gamma \in W^{(2 k-1)}(\beta)$. Now, $W^{(2 k-1)}(\beta)=\bar{H} g \cdot \bar{P} g \cdots \bar{P} g$ $(2 k-1$ copies of $\bar{P} g)$. Thus $\bar{H} g \cdot \bar{P} g \ldots \bar{P} g=\bar{H} f^{-1}$, or

$$
g \bar{P} \cdots g \bar{P} g f \in \bar{H}
$$

or for some $a_{1}, a_{2}, \ldots, a_{2 k-1}$,

$$
g \bar{p}\left(a_{1}\right) g \bar{p}\left(a_{2}\right) \cdots g \bar{p}\left(a_{2 k-1}\right) g f \in \bar{H} .
$$


Note that the $a_{i}$ are nonzero since there can be no returns. So let us look at

$$
\begin{aligned}
g(y) & p\left(a_{1}\right) \cdots g(y) p\left(a_{2 k-1}\right) g(y) f(y) \\
= & r^{(2 k-1)}(y) \cdot\left[\begin{array}{cc}
-y & y^{2} \\
0 & -y
\end{array}\right] \\
= & {\left[\begin{array}{cc}
y^{k+1}\left(-s_{2 k-1}(y)\right) & y^{k+1}\left(y s_{2 k-1}(y)-s_{2 k-2}(y)\right) \\
y^{k}\left(-t_{2 k-1}(y)\right) & y^{k+1}\left(t_{2 k-1}(y)-t_{2 k-2}(y)\right)
\end{array}\right] }
\end{aligned}
$$

based on Lemma 3.5(a), (c), (h), (j). We will use repeatedly throughout this proof the fact that, since $q>k$, two polynomials of degree $k$ or smaller are constant multiples of one another if they have the same roots. Noting that plugging in each value of $y_{i}$ in for $y$ makes this, coordinate-wise, an element of $\bar{H}$, we can conclude the following:

1. The row 1, column 2 entry of (3.6) must be zero for each value of $y_{i}$. Since $\operatorname{deg}\left(y s_{2 k-1}(y)-s_{2 k-2}(y)\right)=k$, this means that for some constant $c_{1}$, we have

$$
c_{1}\left(y s_{2 k-1}(y)-s_{2 k-2}(y)\right)=u_{2 k+2}(y) .
$$

Note that, by Lemma 3.5(f), the constant term on the left is $-c_{1}(-1)^{k-1}=$ $c_{1}(-1)^{k}$, and on the right the constant term is $(-1)^{k}\left(\begin{array}{c}2 k+1-k \\ k\end{array}\right)=(-1)^{k}(k+1)$, and so $c_{1}=k+1$, giving us

$$
(k+1)\left(y s_{2 k-1}(y)-s_{2 k-2}(y)\right)=u_{2 k+2}(y) .
$$

Note that comparing leading coefficients gives us

$$
(k+1) a_{1} \cdots a_{2 k-1}=1 .
$$

2. The ratio of the row 1 , column 1 entry over the row 2 , column 2 entry in (3.6) must be constant for all $y_{i}$. Thus for some constant $c_{2}$, we have

$$
\frac{y^{k+1}\left(-s_{2 k-1}(y)\right)}{y^{k+1}\left(t_{2 k-1}(y)-t_{2 k-2}(y)\right)}=c_{2} .
$$

Hence

$$
c_{2}\left(t_{2 k-1}(y)-t_{2 k-2}(y)\right)+s_{2 k-1}(y)=0 .
$$

Note that the left-hand side of (3.9) is at most a degree $k-1$ polynomial in $y$ $\left(s_{2 k-1}(y), t_{2 k-1}(y)\right.$ are degree $k-1, t_{2 k-2}(y)$ is degree $\left.k-2\right)$ with at least $k$ distinct roots, and so the left-hand side of (3.9) must be identically 0, i.e., every coefficient is 0 . Furthermore, the leading coefficient of $s_{2 k-1}(y)$ is $a_{1} a_{2} \cdots a_{2 k-1}$, and the leading coefficient of $c_{2} t_{2 k-1}(y)$ is $-c_{2} a_{2} a_{3} \cdots a_{2 k-1}$. Since all the $a_{i}$ are nonzero (again, no returns and also (3.8)), we have $c_{2}=a_{1}$, and so

$$
s_{2 k-1}(y)+a_{1} t_{2 k-1}(y)-a_{1} t_{2 k-2}(y)=0 .
$$


3. Finally, the ratio of the row 2, column 1 entry over the row 1 , column 1 entry in (3.6) must be constant for all $y_{i}$. Thus for some constant $c_{3}$, we have

$$
\frac{y^{k}\left(-t_{2 k-1}(y)\right)}{y^{k}\left(-y s_{2 k-1}(y)\right)}=c_{3} \text {. }
$$

So $-c_{3} y s_{2 k-1}(y)+t_{2 k-1}(y)=0$ must hold for $y=y_{1}, \ldots, y_{k}$. Noting that, by Lemma $3.5(\mathrm{c}),(\mathrm{j}),-c_{3} y s_{2 k-1}(y)+t_{2 k-1}(y)$ is a degree $k$ polynomial in $y$, for some constant $c_{4}$, we get that

$$
c_{4}\left(-c_{3} y s_{2 k-1}(y)+t_{2 k-1}(y)\right)=u_{2 k+2}(y) .
$$

Since, by Lemma 3.5(1), the constant term of $c_{4} t_{2 k-1}(y)$ is $c_{4}(-1)^{k}$ and the constant term of $u_{2 k+2}(y)$ is $(k+1)(-1)^{k}$, we get $c_{4}=k+1$. Now, the leading coefficient of $-(k+1) c_{3} y s_{2 k-1}(y)$ is $-(k+1) c_{3} a_{1} \cdots a_{2 k-1}$, and the leading coefficient of $u_{2 k+2}(y)$ is $1=(k+1) a_{1} \cdots a_{2 k-1}$ (from (3.8)), so $c_{3}=-1$, finally giving us

$$
(k+1)\left(y s_{2 k-1}(y)+t_{2 k-1}(y)\right)=u_{2 k+2}(y) .
$$

Comparing (3.7) and (3.11), we get that

$$
s_{2 k-2}(y)=-t_{2 k-1}(y) .
$$

Using Lemma 3.5(c), (j), the leading coefficient of $t_{2 k-1}(y)$ is $-a_{2} \cdots a_{2 k-1}$, and the leading coefficient of $-s_{2 k-2}(y)$ is $-a_{1} a_{2} \cdots a_{2 k-2}$, which means that

$$
a_{2 k-1}=a_{1}
$$

Now, from (3.10), since $t_{2 k-1}(y)=-s_{2 k-2}(y)$, we get

$$
s_{2 k-1}(y)-a_{1} s_{2 k-2}(y)=a_{1} t_{2 k-2}(y) .
$$

Noting (3.12), from part (d) of Lemma 3.5 we get

$$
s_{2 k-1}(y)-a_{1} s_{2 k-2}(y)=-s_{2 k-3}(y) .
$$

Combining these together, we get

$$
s_{2 k-3}(y)=-a_{1} t_{2 k-2}(y) .
$$

The leading coefficient of $-s_{2 k-3}(y)$ is $-a_{1} a_{2} \cdots a_{2 k-3}$, and the leading coefficient of $a_{1} t_{2 k-2}(y)$ is $-a_{1} a_{2} \cdots a_{2 k-3} a_{2 k-2}$, and so we conclude that

$$
a_{2 k-2}=1 \text {. }
$$

We now claim that

$$
\begin{gathered}
a_{1}=a_{3}=a_{5}=\cdots=a_{2 k-3}=a_{2 k-1}, \\
a_{2}=a_{4}=a_{6}=\cdots=a_{2 k-4}=a_{2 k-2}=1 .
\end{gathered}
$$


We will proceed inductively. Assume that for some $i>0$, we have that

$$
\begin{gathered}
a_{2 k-1}=a_{2 k-3}=\cdots=a_{2 k+1-2 i}=a_{1}, \\
a_{2 k-2}=a_{2 k-4}=\cdots=a_{2 k-2 i}=1,
\end{gathered}
$$

and that for all integers $1 \leq j \leq i$, we have

$$
\begin{aligned}
& s_{2 k-2 j}(y)=-t_{2 k-2 j+1}(y), \\
& s_{2 k-2 j-1}(y)=-a_{1} t_{2 k-2 j} .
\end{aligned}
$$

Again using part (d) of Lemma 3.5 and (3.16), we have

$$
\begin{aligned}
s_{2 k-2 i}(y) & =y a_{2 k-2 i} s_{2 k-2 i-1}(y)-s_{2 k-2 i-2}(y) \\
& =y s_{2 k-2 i-1}(y)-s_{2 k-2 i-2}(y) .
\end{aligned}
$$

From part (k) of Lemma 3.5, (3.15), (3.17), and (3.18), we have

$$
\begin{aligned}
s_{2 k-2 i}(y) & =-t_{2 k-2 i+1}(y) \\
& =y a_{2 k-2 i+1}\left(-t_{2 k-2 i}(y)\right)+t_{2 k-2 i-1}(y) \\
& =y a_{1}\left(-t_{2 k-2 i}(y)\right)+t_{2 k-2 i-1}(y) \\
& =y s_{2 k-2 i-1}(y)+t_{2 k-2 i-1}(y) .
\end{aligned}
$$

Comparing (3.19) and (3.20), we get

$$
s_{2 k-2 i-2}(y)=-t_{2 k-2 i-1}(y) \text {. }
$$

The leading coefficient of $s_{2 k-2 i-2}(y)$ is $a_{1} a_{2} \cdots a_{2 k-2 i-2}$, and the leading coefficient of $-t_{2 k-2 i-1}$ is $a_{2} \cdots a_{2 k-2 i-2} a_{2 k-2 i-1}$, and so we conclude that

$$
a_{2 k-2 i-1}=a_{1} \text {. }
$$

Again using part (d) of Lemma 3.5 and (3.22), we have

$$
\begin{aligned}
s_{2 k-2 i-1}(y) & =a_{2 k-2 i-1} s_{2 k-2 i-2}(y)-s_{2 k-2 i-3}(y) \\
& =a_{1} s_{2 k-2 i-2}(y)-s_{2 k-2 i-3}(y) .
\end{aligned}
$$

From part (k) of Lemma 3.5, (3.16), (3.18), and (3.21), we have

$$
\begin{aligned}
s_{2 k-2 i-1}(y) & =a_{1}\left(-t_{2 k-2 i}(y)\right) \\
& =a_{1}\left(a_{2 k-2 i}\left(-t_{2 k-2 i-1}(y)\right)+t_{2 k-2 i-2}(y)\right) \\
& =a_{1}\left(-t_{2 k-2 i-1}(y)\right)+a_{1} t_{2 k-2 i-2}(y) \\
& =a_{1} s_{2 k-2 i-2}(y)+a_{1} t_{2 k-2 i-2}(y) .
\end{aligned}
$$


Comparing (3.23) and (3.24), we get

$$
s_{2 k-2 i-3}(y)=-a_{1} t_{2 k-2 i-2}(y) .
$$

The leading coefficient of $s_{2 k-2 i-3}(y)$ is $a_{1} a_{2} \cdots a_{2 k-2 i-3}$, and the leading coefficient of $-a_{1} t_{2 k-2 i-2}$ is $a_{1} a_{2} \cdots a_{2 k-2 i-3} a_{2 k-2 i-2}$, and so we conclude that

$$
a_{2 k-2 i-2}=1
$$

Therefore, by induction, we can continue this process indefinitely, and thus (3.13) and (3.14) must hold. Going back to (3.8), we have $(k+1) a_{1} \cdots a_{2 k-1}=1$, and so $(k+1) a_{1}^{k}=1$, or

$$
a_{1}^{k}=\frac{1}{k+1} .
$$

Now, we go back to $(3.7),(k+1) y s_{2 k-1}(y)-(k+1) s_{2 k-2}(y)=u_{2 k+2}(y)$, and look at the coefficient of $y^{k-1}$. From part (e) of Lemma 3.5, the coefficient of $y^{k-1}$ in $(k+1) y s_{2 k-1}(y)$ is

$$
-(k+1)(2 k-2) a_{1}^{k-1} .
$$

The coefficient of $y^{k-1}$ in $-(k+1) s_{2 k-2}(y)$ is just the leading coefficient, which is

$$
-(k+1) a_{1} \cdots a_{2 k-2}=-(k+1) a_{1}^{k-1} .
$$

Finally, the coefficient of $y^{k-1}$ in $u_{2 k+2}(y)$ is

$$
(-1)^{1}\left(\begin{array}{c}
2 k+1-1 \\
1
\end{array}\right)=-2 k .
$$

Noting that

$$
a_{1}^{k-1}=\frac{1}{a_{1}(k+1)}
$$

and equating the coefficient of $y^{k-1}$ on each side of (3.7), we have

$$
\frac{2 k-2}{a_{1}}+\frac{1}{a_{1}}=2 k,
$$

or

$$
a_{1}=\frac{2 k-1}{2 k} .
$$

Again, we go back to (3.7), but this time we look at the coefficient of $y$. Using part (f) of Lemma 3.5 and then (3.26), the linear term of $(k+1) y s_{2 k-1}(y)$ is

$$
\begin{aligned}
(k+1)(-1)^{k-1}\left(a_{1}+a_{3}+\cdots+a_{2 k-1}\right) & =(k+1)(-1)^{k-1}\left(k a_{1}\right) \\
& =(-1)^{k-1}(k+1) \frac{2 k-1}{2} .
\end{aligned}
$$


From part (g) of Lemma 3.5 and (3.26), the linear term of $(k+1) s_{2 k-2}(y)$ is

$$
(-1)^{k}(k+1) \frac{(k-1) k}{2} \frac{2 k-1}{2 k}=(-1)^{k} \frac{(k+1)(k-1)(2 k-1)}{4} .
$$

Finally, the linear term in $u_{2 k+2}(y)$ is

$$
(-1)^{k-1}\left(\begin{array}{c}
2 k+1-k+1 \\
k-1
\end{array}\right)=(-1)^{k-1} \frac{(k+2)(k+1) k}{6} .
$$

Putting these together, from (3.7) we get

$$
\frac{(k+1)(2 k-1)}{2}+\frac{(k+1)(k-1)(2 k-1)}{4}=\frac{(k+2)(k+1) k}{6},
$$

which simplifies to

$$
(4 k+3)(k-1)=0 .
$$

Case 1. If $k-1=0$, then $k=1$ in $\operatorname{GF}(q)$. We know that

$$
a_{1}=\frac{2 k-1}{2 k}=\frac{1}{2}
$$

in $\mathrm{GF}(q)$. From (3.25) we get that

$$
\begin{aligned}
& (k+1) a_{1}^{k}=1, \\
& (2)\left(\frac{1}{2}\right)^{k}=1, \\
& 2^{k-1}-1=0 .
\end{aligned}
$$

Thus we must have $p|(k-1), p|\left(2^{k-1}-1\right)$.

Case 2. If $4 k+3=0$, then $k=-\frac{3}{4}$ in $\operatorname{GF}(q)$. We know that

$$
a_{1}=\frac{2 k-1}{2 k}=\frac{5}{3}
$$

in $\operatorname{GF}(q)$. Again from (3.25) we get

$$
\begin{aligned}
& \left(\frac{1}{4}\right)\left(\frac{5}{3}\right)^{k}=1, \\
& 5^{k}-4 \cdot 3^{k}=0 .
\end{aligned}
$$

Thus we must have $p|(4 k+3), p|\left(5^{k}-4 \cdot 3^{k}\right)$.

We now can easily prove the main results. 
Proof of Theorem 1.1 For a given even $m=2 k+2$, we construct our infinite family of near-polygonal graphs with special cycles of length $m$ as in Lemma 3.4. By Lemma 3.6 these graphs all have girth at least $m-1$, and by Theorem 3.7 for only finitely many primes $p$ can the constructed graph have girth $m-1$. Hence there are infinitely many 2-arc transitive polygonal graphs of girth $m$ for all even $m \geq 4$.

Proof of Corollary 1.2 Combining the results of [4, Theorem 1.1] and Theorem 1.1 proves this result.

\section{References}

1. Li, C.H., Seress, A.: Symmetrical path-cycle covers of a graph and polygonal graphs. J. Comb. Theory A 114(1), 35-51 (2007)

2. Perkel, M.: Near-polygonal graphs. Ars. Comb. A 26, 149-170 (1988)

3. Seress, A.: Polygonal graphs. In: Horizons of Combinatorics. Bolyai Society Mathematical Studies, vol. 17, pp. 179-188. Springer, Berlin (2008). Chap. 9

4. Swartz, E.: A construction of an infinite family of 2-arc transitive polygonal graphs of arbitrary odd girth. J. Comb. Theory, Ser. A 117(6), 783-789 (2010) 